\title{
Numerical simulation on the influences of Wenchuan earthquake on the surrounding faults*
}

\author{
Yujiang $\mathrm{Li}^{*}$ Lianwang Chen and Yuanzhong Lu \\ Key Laboratory of Crustal Dynamics, Institute of Crustal Dynamics, China Earthquake \\ Administration, Beijing 100085, China
}

\begin{abstract}
On 12 May 2008, the devastating Wenchuan earthquake struck the Longmenshan fault zone, which comprised the eastern margin of the Tibetan Plateau, and this fault zone was predominantly a convergent boundary with a right-lateral strike-slip component. After such a large-magnitude earthquake, it was crucial to analyze the influences of the earthquake on the surrounding faults and the potential seismic activity. In this paper, a complex viscoelastic model of western Sichuan and eastern Tibet regions was constructed including the topography. Based on the findings of co-seismic static slip distribution, we calculated the stress change caused by the Wenchuan earthquake with the post-seismic relaxation into consideration. Our preliminary results indicated that: (1) The tectonic stressing rate was relatively high in Kunlun mountain pass-Jiangcuo, Ganzi-Yushu, Xianshuihe and Zemuhe faults; while in the east Kunlun and Longriba was medium; also the value was less in the Minjiang, Longmenshan, Anninghe and Huya faults. As to the Longmenshan fault, the value was $0.28 \times 10^{-3} \mathrm{MPa} / \mathrm{a}$ to $0.35 \times 10^{-3} \mathrm{MPa} / \mathrm{a}$, which is coincident with the previous long recurrence interval of Wenchuan earthquake; (2) The Wenchuan earthquake not only caused the Coulomb stress decrease in the source region, but also the stress increase in the two terminals, especially the northeastern segment, which is comparatively consistent with the aftershock distribution. Meanwhile, the high concentration areas of the static slip distribution were corresponding to the Coulomb stress reductions; (3) The Coulomb stress change caused by Wenchuan earthquake showed significant increase on five major faults, which were northwestern segment of Xianshuihe fault, eastern Kunlun fault, Longriba fault, Minjiang fault and Huya fault respectively; also the Coulomb stress on the fault plane of the Yushu earthquake was faintly increased; (4) We defined the recurrence interval as the time needed to accumulate the magnitude of the stress drop, and the recurrence interval of Wenchuan earthquake was estimated about 1714 a to 2143 a correspondingly.
\end{abstract}

Key words: Longmenshan fault zone; Wenchuan earthquake; Coulomb failure stress; tectonic stressing rate; numerical simulation

CLC number: P315.72+7 Document code: A

\section{Introduction}

On 12 May 2008, the devastating Wenchuan earthquake struck the Longmenshan fault zone about $300 \mathrm{~km}$ (Densmore et al., 2007; Burchfiel et al., 2008), which comprised the eastern margin of the Tibetan Plateau, collapsing buildings and killing thousands in major cities

\footnotetext{
* Received 6 January 2012; accepted in revised form 20 March 2012; published 10 April 2012.

* Corresponding author. e-mail: toleeyj@126.com

(C) The Seismological Society of China, Institute of Geophysics, China Earthquake Administration, and Springer-Verlag Berlin Heidelberg 2012
}

of Sichuan province, China. Preliminary teleseismic waveform analysis suggested that the earthquake was composed of two sub-events of 6-9 m slip on an about $33^{\circ}$ dipping fault, the epicentral sub-event underwent oblique right-lateral thrust slip, while the northeast subevent slipped largely right-laterally (Nishimura and Yagi, 2008; Ji and Hayes, 2008). After such an earthquake, it was crucial to analyze the stress transfer in the crust. Then, we employed the finite element model to study the stress change caused by Wenchuan earthquake with the post-seismic relaxation into consideration.

In the history records, there were many earth- 
quakes in the Longmenshan adjacent area, such as the Diexi M7.3 in 1933 (Kan et al., 1977), the SongpanPingwu M7.2 double shocks in 1966 (Molnar and Deng, 1984), which were about one hundred kilometers to this fault. However, in the last three hundred years, there was no $M_{\mathrm{S}}>6.0$ earthquake in the Longmenshan fault system. Unexpectedly, the Wenchuan earthquake occurred on this fault which was seemingly inactive from the GPS observation before.

\section{Finite element model}

Based on the latest achievements about active tectonics, velocity structure in western Sichuan and eastern Tibet regions, a finite element model is constructed to simulate the stress change following the Wenchuan earthquake. The model incorporates five key features: (1) The boundary is determined as follows: $26^{\circ} \mathrm{N}-37^{\circ} \mathrm{N}$ in latitude and $90^{\circ} \mathrm{E}-108^{\circ} \mathrm{E}$ in longitude, also the depth reaches $100 \mathrm{~km}$; (2) The model includes five layers with the topography into consideration, and all these fault$\mathrm{s}$ in the model are simulated by the frictional contact, that is to say, contact elements have zero thickness; (3) The faults in the model are discontinuities and have deformable contact elements on their surface that obey the Coulomb failure criterion; (4) The Longmenshan fault zone has a high dipping angle near surface and low-angle at depth, and this kind of listric shape favors significant strain to form great earthquakes; (5) The crustal velocity structure and density are known in advance (Wang et al., 2007; Huang et al., 2003; Zhu, 2008), and the elastic parameters can be inferred (Table 1), the viscosity follows the previous results (Shi and Cao, 2008; Zhang et al., 2008a).

\subsection{Model description}

The finite element model is composed entirely of eight-node viscoelastic elements (Figure 1). It consists of 135547 elements with 141527 active nodes. The surface elevation is mainly based on the SRTM (Shuttle Radar Topography Mission) elevation data (http://srtm.csi.cgiar.org/). Since the Longmenshan fault zone has a high dipping angle near surface and low-angle at depth, we use the listric shape to simulate the fracture surface morphology, and this kind of shape favors significant strain or energy accumulation to form great earthquakes (Zhu and Zhang, 2009). As the earthquake preparation process generally takes several hundred to several thousand years and even longer, to a certain extent, the rock stress-strain accumulation presents the rheological properties. Therefore, it is necessary to consider the rheological properties of the medium.

Table 1 Material properties of the crust and upper mantle

\begin{tabular}{|c|c|c|c|c|c|}
\hline Layer & Depth/km & Partition & $\begin{array}{c}\text { Young's modulus } \\
\text { /GPa }\end{array}$ & $\begin{array}{c}\text { Poisson's ratio } \\
\nu\end{array}$ & $\begin{array}{c}\text { Viscosity } \\
/\left(10^{19} \mathrm{~Pa} \cdot \mathrm{s}\right)\end{array}$ \\
\hline \multirow{2}{*}{ Surface } & \multirow{2}{*}{$0-5$} & 1 & 60.8 & 0.25 & 100 \\
\hline & & 2 & 20.3 & 0.25 & 100 \\
\hline \multirow{6}{*}{ Upper crust } & \multirow{6}{*}{$5-16$} & 3 & 81.0 & 0.25 & 100 \\
\hline & & 4 & 83.7 & 0.25 & 100 \\
\hline & & 5 & 86.5 & 0.25 & 100 \\
\hline & & 6 & 27.0 & 0.26 & 100 \\
\hline & & 7 & 2.74 & 0.26 & 100 \\
\hline & & 8 & 2.90 & 0.26 & 100 \\
\hline \multirow{6}{*}{ Middle crust } & \multirow{6}{*}{$16-30$} & 9 & 7.70 & 0.26 & 1 \\
\hline & & 10 & 8.97 & 0.25 & 10 \\
\hline & & 11 & 9.56 & 0.25 & 10 \\
\hline & & 12 & 2.57 & 0.28 & 1 \\
\hline & & 13 & 2.77 & 0.28 & 1 \\
\hline & & 14 & 2.88 & 0.28 & 1 \\
\hline \multirow{6}{*}{ Lower crust } & \multirow{6}{*}{$30-65$} & 15 & 10.5 & 0.28 & 10 \\
\hline & & 16 & 11.6 & 0.26 & 10 \\
\hline & & 17 & 14.4 & 0.26 & 10 \\
\hline & & 18 & 3.50 & 0.30 & 10 \\
\hline & & 19 & 3.67 & 0.30 & 10 \\
\hline & & 20 & 4.33 & 0.30 & 10 \\
\hline \multirow{6}{*}{ Upper mantle } & \multirow{6}{*}{$65-100$} & 21 & 14.9 & 0.30 & 10 \\
\hline & & 22 & 16.5 & 0.28 & 10 \\
\hline & & 23 & 18.6 & 0.26 & 10 \\
\hline & & 24 & 4.97 & 0.35 & 10 \\
\hline & & 25 & 5.23 & 0.35 & 10 \\
\hline & & 26 & 5.57 & 0.35 & 10 \\
\hline
\end{tabular}




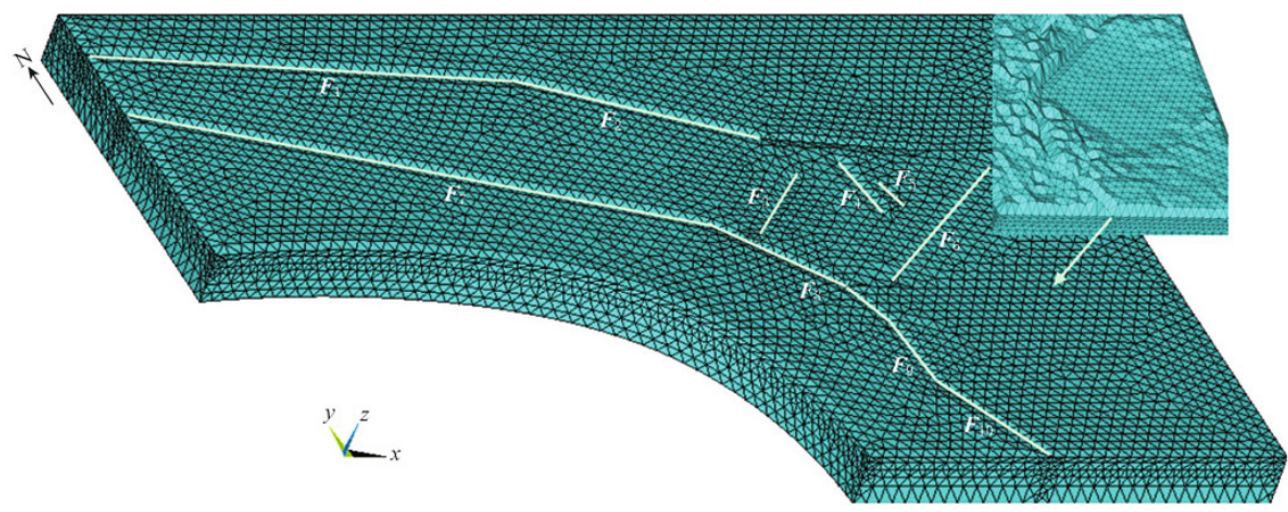

Figure 1 Three dimensional discretization model. $F_{1}$ to $F_{10}$ represent the Kunlun mountain pass-Jiangcuo, eastern Kunlun, Longriba, Minjiang, Huya, Longmenshan, Ganzi-Yushu, Xianshuihe, Anninghe and Zemuhe faults, respectively, and the geometric parameters are in details in Table 2 according to the recent findings (Xu et al., 2008), while the right is the digital elevation increased by five times around the Sichuan basin, and the elevation difference was obvious.

Table 2 Geometric parameters of the major active faults

\begin{tabular}{ccccc}
\hline Fault & Strike & Dip & Inclination & Activity \\
\hline Xianshuihe fault (NW) & $\mathrm{N} 40^{\circ} \mathrm{W}$ & $\mathrm{NE} / \mathrm{SW}$ & vertical & $\mathrm{LL}$ \\
Xianshuihe fault (SE) & $\mathrm{N} 20^{\circ} \mathrm{W}$ & $\mathrm{NE} / \mathrm{SW}$ & vertical & $\mathrm{LL}$ \\
Kunlun mountain pass fault & $\mathrm{EW}$ & $\mathrm{N} / \mathrm{S}$ & vertical & $\mathrm{LL}+\mathrm{R}$ \\
Ganzi-Yushu fault & $\mathrm{NW}$ & $\mathrm{NE}$ & vertical & $\mathrm{LL}$ \\
Kunlun fault & $\mathrm{WNW}$ & $\mathrm{NE} / \mathrm{SW}$ & vertical & $\mathrm{LL}+\mathrm{R}$ \\
Longmenshan fault & $\mathrm{N} 45^{\circ} \mathrm{E}$ & $\mathrm{NW}$ & $65^{\circ}$ (up), $25^{\circ}$ (down) & $\mathrm{RL}+\mathrm{R}$ \\
Anninghe fault & $\mathrm{NS}$ & $\mathrm{E} / \mathrm{W}$ & vertical & $\mathrm{LL}+\mathrm{R}$ \\
Zemuhe fault & $\mathrm{N} 25^{\circ} \mathrm{W}$ & $\mathrm{SW}$ & vertical & $\mathrm{LL}+\mathrm{R}$ \\
Longriba fault & $\mathrm{N} 60^{\circ} \mathrm{E}$ & $\mathrm{NW}$ & vertical & $\mathrm{RL}$ \\
Minjiang fault & $\mathrm{NS}$ & $\mathrm{E} / \mathrm{W}$ & vertical & vertical $+\mathrm{R}$ \\
Huya fault & $\mathrm{NNW}$ & $\mathrm{E} / \mathrm{W}$ & $\mathrm{LL}$ \\
\hline
\end{tabular}

Note: LL presents left-lateral strike-slip, RL presents right-lateral strike-slip, and R presents reverse dip-slip.

Among the previous researches, the Maxwell body or the power-law fluid was widely used in the earthquake-related fields (Zhang et al., 2009; Wu et al., 2011), the relaxation and creep characteristics of the Maxwell body have shown a similar fluid nature property, in which the stress reduces to zero, or the strain increases infinitely with time, and this may not match the crustal rheological characteristics. As a nonlinear constitutive relation, the power-law fluid needs the higher convergence value. In the end, we choose the standard linear solid constitutive in this paper. And the creep and relaxation equations of this constitutive relation are

$$
\varepsilon=\frac{\sigma_{0}}{q_{0}}\left[1-\left(1-\frac{p_{1} q_{0}}{q_{1}}\right) \mathrm{e}^{-t / \tau}\right],
$$

and

$$
\sigma=q_{0} \varepsilon_{1}\left(1-e^{-t / p_{1}}\right)+\frac{q_{1}}{p_{1}} \varepsilon_{1} \mathrm{e}^{-t / p_{1}},
$$

where $p_{1}=\eta /\left(E_{1}+E_{2}\right), q_{0}=E_{1} \cdot E_{2} /\left(E_{1}+E_{2}\right), q_{1}=E_{1} \cdot \eta /$ $\left(E_{1}+E_{2}\right), \tau=q_{1} / q_{0}=\eta / E_{2}, E_{1}, E_{2}$ represent the Young's modulus and $\eta$ the viscosity.

As we all know, the Maxwell body shows a similar fluid properties, and as a solid medium with the rheological properties, the standard linear solid is more realistic than the Maxwell or Kelvin body, and the creep and relaxation characteristics are shown in Figure 2. For the creep equation, the strain increases to a constant with the invariable stress, not the infinite; while for the relaxation equation, the stress reduces to a constant with the invariable strain, not zero.

\subsection{Boundary conditions and loads}

GPS velocity observations are interpolated and extrapolated across the model and boundary condition areas, and the model is loaded according to a thousand year displacements, then the tectonic stressing rate is evaluated. Meanwhile, the model base is freely 

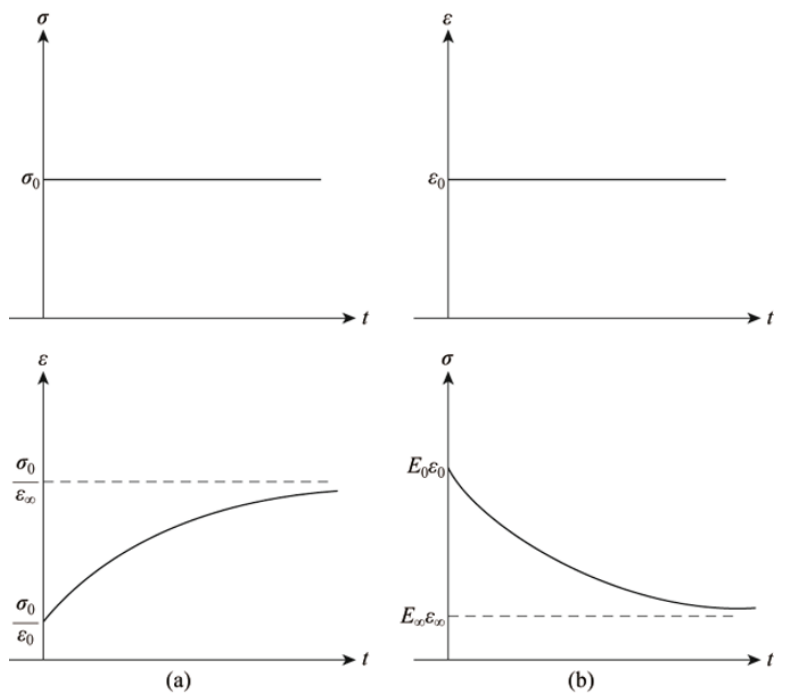

Figure 2 Creep (a) and relaxation (b) curves of the standard linear solid body where $E_{0}$ and $E_{\infty}$ represent the Young's modulus in the initial and the infinite time, while $\varepsilon_{0}$ and $\varepsilon_{\infty}$ represent the strain in the same condition, $\sigma_{0}$ represents the initial stress.

slipping laterally but cannot move vertically, and the model free surface is fully deformable. All constraints are imposed on the model as described above; before any constrain is introduced, the model is subjected to gravity for a ten thousand years period required to fully compress under its own weight.

All modeling presented here is conducted using the ANSYS finite element program. ANSYS employs the Newton-Raphson approach to solve nonlinear problems. In this method a load is subdivided into a series of increments applied over several steps. Before each solution this method evaluates the out-of-balance load vector. If the convergence criteria are not satisfied, the load vector is reevaluated, the stiffness matrix is updated, and a new solution is obtained until the problem converges.

\section{Coulomb failure stress change results}

An earthquake can be modeled as an elastic dislocation solutions in an elastic half-space (Okada, 1992), which makes the estimation of stress transfer and prediction of the aftershock distribution available immediately after an earthquake, and it is widely used nowadays (Harris, 1998; Stein, 1999; Gomberg and Felzer, 2008). When the Wenchuan earthquake occurred, numerous Coulomb failure stress studies have been carried out. Parsons et al. (2008) calculated the stress change in the vicinity of the Longmenshan fault zone and estimated the earthquake probability. Toda et al. (2008) did a similar analysis to forecast the seismic rate and distribution of damaging shocks using the calculated stress changes and the observed background seismicity. Wan and Shen (2010) used an updated earthquake source model and the fault geometry as well as kinematic parameters to attempt a better assessment of the earthquake potentials in the region, also to evaluate the Coulomb failure stress assuming different effective friction coefficients; the results are in general agreements with previous studies. Shan et al. (2009) used the PSGRN/PSCMP software and the Crust 2.0 model to study the stress change caused by Wenchuan earthquake, and also the uncertainty deduced from the effective friction coefficient. Unfortunately, the shortcomings such as the coupled viscoelastic relaxation effect and inhomogeneous medium treatment were obvious (Nur and Mavko, 1974), the initial stress was also not included. However, the initial stress not only made the fault plane stress state more complex, but also affected the distribution of Coulomb stress (King et al., 1994), while the finite element method was relatively flexible.

Calculated changes in stress tensor components are resolved on the interest planes, and changes in failure stress are related to triggering or inhibition of future earthquakes (Chen et al., 2008). Usually, the Coulomb stress change is used to explain patterns of seismicity (Yamashina, 1978; Stein, 1999). The Coulomb failure criterion is defined as

$$
\Delta \sigma_{\mathrm{f}}=\Delta \tau+\mu\left(\Delta \sigma_{\mathrm{n}}+\Delta p\right)
$$

where $\Delta \tau$ is the shear stress acting on the receiver fault (positive in the fault slip direction), $\mu$ is the friction coefficient, $\Delta \sigma_{\mathrm{n}}$ is the change in normal stress acting on the receiver fault (positive for unclamping), $\Delta p$ is the pore pressure change. The effect of friction reduction due to pore pressure can be represented by an equivalent friction coefficient $\mu^{\prime}=\mu(1-B)$, in which $B$ is the Skempton coefficient in the range of 0-1 (Rice, 1992), then $\Delta \sigma_{\mathrm{f}}=\Delta \tau+\mu^{\prime} \Delta \sigma_{\mathrm{n}}$, also in our calculation, we are assuming the equivalent friction coefficient value of $\mu^{\prime}=0.4$.

\subsection{Tectonic stressing rate}

Tectonic stressing rate is simulated on the finite element model with the GPS constraints, and this period lasts one thousand years. After one thousand years the Wenchuan earthquake is simulated by displacing the Longmenshan fault with the slip distribution of $\mathrm{Ji}$ 
and Hayes (2008). From Figure 3 we can conclude that the stressing rate is relatively high in Kunlun mountain pass-Jiangcuo, Ganzi-Yushu, Xianshuihe and Zemuhe faults; while the east Kunlun and Longriba is medium; also the value is less in the Minjiang, Longmenshan, Anninghe and Huya faults. As to the Longmenshan fault, the value is $0.28 \times 10^{-3} \mathrm{MPa} / \mathrm{a}$ to $0.35 \times 10^{-3} \mathrm{MPa} / \mathrm{a}$, which is coincident with the previous long recurrence interval of Wenchuan earthquake (Densmore et al., 2007; Burchfiel et al., 2008; Zhang et al., 2008b; Shen et al., 2009).

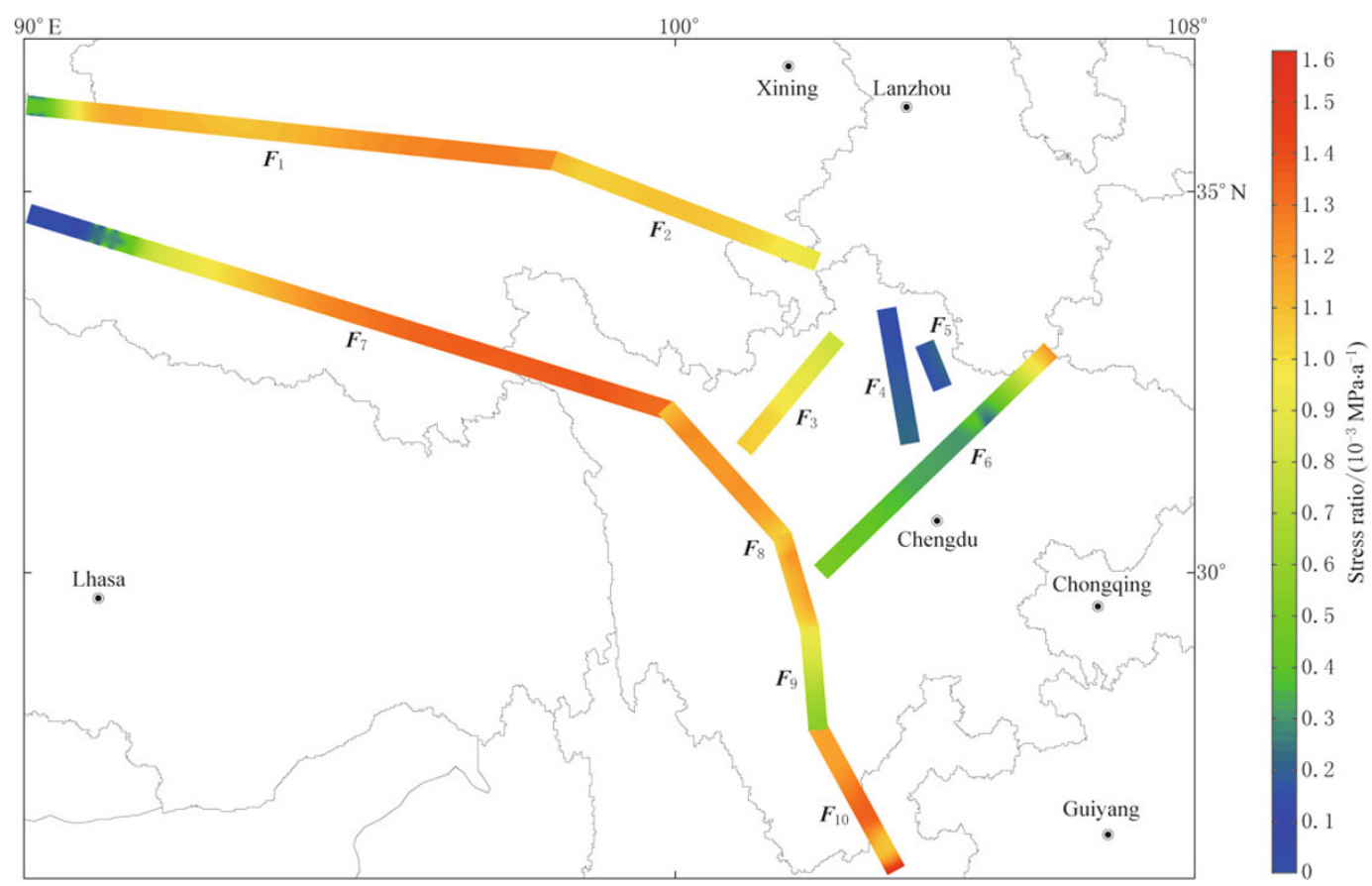

Figure 3 Tectonic stressing rate on the mainly active faults in the upper crust. $F_{1}$ to $F_{10}$ represent the Kunlun mountain pass-Jiangcuo, eastern Kunlun, Longriba, Minjiang, Huya, Longmenshan, Ganzi-Yushu, Xianshuihe, Anninghe and Zemuhe faults, respectively.

\subsection{Coulomb failure stress changes caused by Wenchuan earthquake}

The Coulomb failure stress change on a receiver fault not only depends on the fault geometry (strike, dip and rake) and friction coefficient, but also the geometry of the source fault, as well as the coseismic slip of the source earthquake (King et al., 1994). Before the findings of co-seismic static slip distribution was introduced (Figure 4), the model was subjected to gravity for a ten-thousand-year period required to fully compress under its own weight, then with the post-seismic relaxation into consideration, the Coulomb failure stress change caused by the Wenchuan earthquake was examined in detail, as shown in Figure 5.

The Wenchuan earthquake not only caused the Coulomb failure stress decrease in the source region, but also the stress increase in the two terminals, especially the northeastern segment, which is comparatively consistent with the aftershock distribution. Meanwhile, the high concentration area of the static slip distribution was corresponding to the stress reduction; the Coulomb failure stress change caused by Wenchuan earthquake was significantly increased on five major fault segments, which were northwestern segment of Xianshuihe fault, eastern Kunlun fault, Longriba fault, Minjiang fault and Huya fault respectively, also the Coulomb failure stress on the fault plane of the Yushu earthquake was faintly increased. The earthquake also decreased the Coulomb failure stress on the southeastern segment of Xianshuihe fault, Anninghe fault, especially on the southeastern

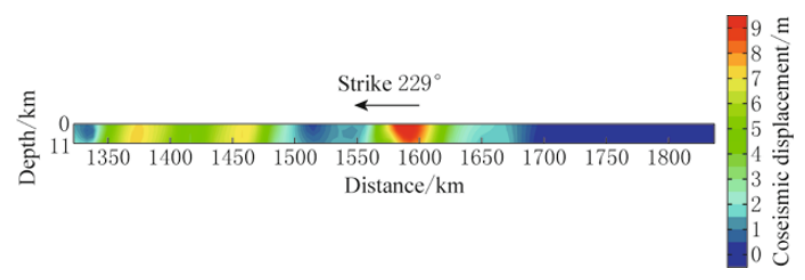

Figure 4 Distribution of the coseismic displacement in the seismogenic fault (After Ji and Hayes, 2008). 


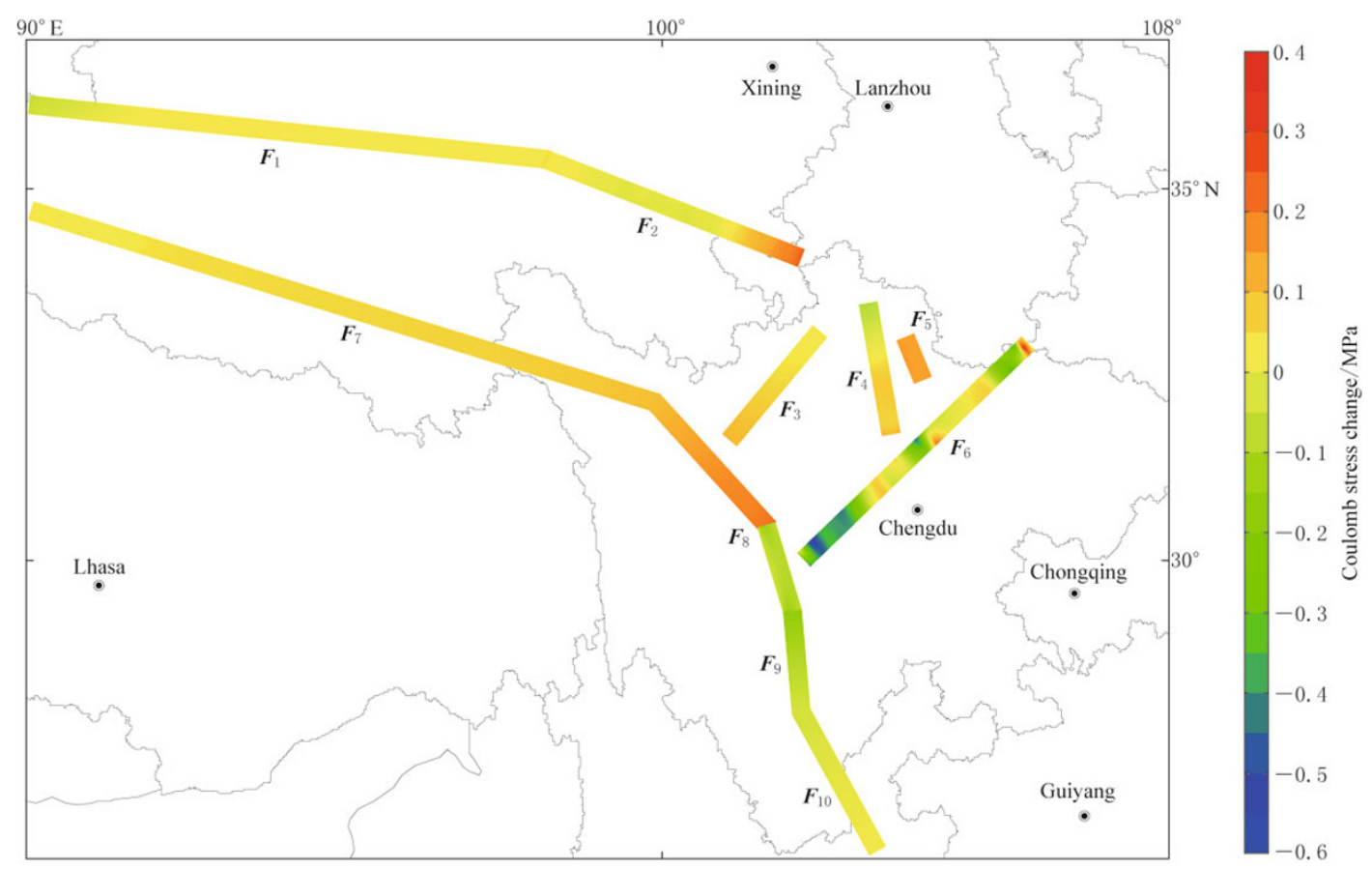

Figure 5 Coulomb stress change on major faults caused by the Wenchuan earthquake. The fault names are the same as those in Figure 3.

segment of Xianshuihe fault with the stress reduction of $0.3 \mathrm{MPa}$, respectively. The Coulomb failure stress change on the fault plane of Zemuhe was nearly zero, indicating no significant influence of the Wenchuan earthquake on the fault. In short, the Coulomb stress change was mainly increasing in the area north of the Longmenshan fault zone while the south was different degrees of reduction.

\subsection{Estimation of the recurrence interval in the Longmenshan fault zone}

From the point of the excavation trenches after the earthquake, both the central and frontier-range faults of the Longmenshan had shown at least two seismic events with the size of the Wenchuan earthquake, and the preliminary results were of characteristic earthquake type (Ran et al., 2008; Zheng et al., 2008). We defined the recurrence interval as the time needed to accumulate the magnitude of the stress drop. In line with our result of the tectonic stressing rate in Figure 3, the recurrence interval of Wenchuan earthquake in the Longmenshan fault was estimated about 1714 a to 2143 a correspondingly, which was comparatively consistent with the other methods, such as the paleoseismic evidence, fault slip rate, earthquake moment rate and GPS observation data (Zhang et al., 2008b; Xie et al., 2008; Ren et al., 2009; Zhu and Zhang, 2009; Ran et al., 2010).

\section{Conclusions}

The 12 May 2008 Wenchuan earthquake caused grievous losses, yet its legacy included possible large shocks in the near future around the Sichuan basin. In view of this point, GPS-derived displacement was used to distort a finite element model of western Sichuan and eastern Tibet, and the tectonic stressing rate was given. Based on the findings of co-seismic static slip distribution deduced from the teleseismic waveform analysis, the Coulomb failure stress change caused by the Wenchuan earthquake was introduced with the post-seismic relaxation into consideration, then the preliminary results showed that the effect was changed in partition in the area north of the Longmenshan fault; the effect was mainly loaded while in the south uploaded. That is to say, if such an earthquake in the future is observed on the stressed parts of the Xianshuihe, east Kunlun, Longriba, Minjiang and Huya faults, it would provide support for hypothesis that large shocks are now also more likely to happen where the static stress imparted by the mainshock has risen. Finally, the recurrence interval of the Longmenshan fault was estimated about 1714 a to 2143 a correspondingly. Furthermore, the postseismic effect such as the afterslip was ignored in this paper, which will be done later. 
Acknowledgements Thanks are given to Professor Yongge Wan and the three anonymous reviewers for their helpful suggestions, also to Yanxing Li and Chen Ji for providing the data. This work was financially supported by Basic Science and Research from the Institute of Crustal Dynamics, China Earthquake Administration (ZDJ2012-09, ZDJ2010-12), and the National Key Technology Research and Development Program (2008BAC38B04).

\section{References}

Burchfiel B C, Royden L H, vander Hilst R D, Hager B H, Chen Z, King R W, Li C, Lü J, Yao H and Kirby E (2008). A geological and geophysical context for the Wenchuan earthquake of 12 May 2008, Sichuan, People's Republic of China. GSA Today 18(7): 4-11, doi:10.1130/GSATG18A.1.

Chen L W, Zhang P Z, Lu Y Z, Chen H R, Ma H S, $\mathrm{Li} \mathrm{L}$ and $\mathrm{Li} \mathrm{H}$ (2008). Numerical simulation of loading/uploading effect on Coulomb failure stress among strong earthquakes in Sichuan-Yunnan area. Chinese $J$ Geophys 51(5): 1411-1 421 (in Chinese with English abstract).

Densmore A L, Ellis M A, Li Y, Zhou R J, Hancock G $\mathrm{S}$ and Richardson N (2007). Active tectonics of the Beichuan and Pengguan faults at the eastern margin of the Tibetan Plateau. Tectonics 26: TC4005, doi:10.1029/2006TC001987.

Gomberg J and Felzer K (2008). A model of earthquake triggering probabilities and application to dynamic deformation constrained by ground motion observations. $J$ Geophys Res 113: B10317, doi:10.1029/2007JB005184.

Harris R A (1998). Introduction to special section, stress triggers, stress shadows, and implication for seismic hazard. J Geophys Res 103: 24 347-24 358.

Huang Z X, Su W, Peng Y J, Zheng Y J and Li H Y (2003). Rayleigh wave tomography of China and adjacent regions. J Geophys Res 108(B2): 2 073, doi:10.1029/2001JB001696.

Ji C and Hayes G (2008). Preliminary result of the May 12, $2008 M_{\mathrm{W}} 7.9$ eastern Sichuan, China earthquake. [2008-07-20]. http://earthquake.usgs.gov/eqcenter/eqinthenews/2008/ us2008ryan/finite_fault.php.

Kan R J, Zhang S C, Yan F T and Yu L S (1977). Present tectonic stress field and its relation to the characteristics of recent tectonic activity in Southwestern China. Chinese J Geophys 20(2): 96-109 (in Chinese with English abstract).

King G C P, Stein R S and Lin J (1994). Static stress changes and the triggering of earthquakes. Bull Seismol Soc Am 84(3): 935-953.

Molnar P and Deng Q (1984). Faulting associated with large earthquakes and the average rate of deformation in cen- tral and eastern Asia. J Geophys Res 89: 6 203-6 228.

Nishimura N and Yagi Y (2008). Rupture process for May 12, 2008 Sichuan earthquake (Ver. 2). [2008-06-12]. http://www.geol.tsukuba.ac.jp/ nisimura/20080512.

Nur A and Mavko G (1974). Postseismic viscoelastic rebound. Science 183: 204-206.

Okada Y (1982). Internal deformation due to shear and tensile faults in a half-space. Bull Seismol Soc Am 82: $1018-1040$.

Parsons T, Ji C and Kirby E (2008). Stress changes from the 2008 Wenchuan earthquake and increased hazard in the Sichuan basin. Nature 454: 509-510, doi:10.1038/nature07177.

Ran Y K, Chen L C, Chen G H, Yin J H, Chen J, Gong H L, Shi X and Li C X (2008). Primary analyses of in-situ recurrence of large earthquake along seismogenic fault of the $M_{\mathrm{S}} 8.0$ Wenchuan earthquake. Seismology and Geology 30(3): 630-642 (in Chinese with English abstract).

Ran Y K, Chen L C, Chen J, Wang H, Chen G H, Yin J H, Shi X, Li C X and Xu X W (2010). Paleoseismic evidence and repeat time of large earthquake at three sites along the Longmenshan fault zone. Tectonophysics 491 : 141-153, doi:10.1016/j.tecto.2010.01.009.

Ren J J, Zhang S M, Ma B Q and Tian Q J (2009). Characteristics and recurrence intervals of large earthquakes along the middle-northern segment of the Longmenshan fault zone. Acta Seismologica Sinica 31(2): 160-171 (in Chinese with English abstract).

Rice J R (1992). Fault stress state, pore pressure distributions and the weakness of the San Andreas fault. In: Evans B and Wong T-F eds. Fault Mechanics and Transport Properties of Rock. Academic, San Diego, Calif, 475503.

Shan B, Xiong X, Zheng Y and Diao F Q (2009). Stress changes on major faults caused by $M_{\mathrm{W}} 7.9$ Wenchuan earthquake, May 12, 2008. Science in China (Series D) 39(5): 537-545 (in Chinese with English abstract).

Shen Z K, Sun J B, Zhang P Z, Wan Y G, Wang M, Bürgmann R, Zeng Y H, Gan W J, Liao H and Wang Q L (2009). Slip maxima at fault junctions and rupturing of barriers during the 2008 Wenchuan earthquake. Nature Geoscience 2: 718-724, doi:10.1038/ngeo636.

Shi Y L and Cao J L (2008). Effective viscosity of China continental lithosphere. Earth Science Frontiers 15(3): 82-95 (in Chinese with English abstract).

Stein R S (1999). The role of stress transfer in earthquake occurrence. Nature 402: 605-609.

Toda S, Lin J, Meghraoui M and Stein R S (2008). 12 May $2008 M=7.9$ Wenchuan, China, earthquake calculated to increase failure stress and seismicity rate on three major fault systems. Geophys Res Lett 35: L17305, doi:10.1029/2008GL034903.

Wan Y G and Shen Z K (2010). Static Coulomb stress changes on faults caused by the $2008 M_{\mathrm{W}} 7.9$ Wenchuan, 
China earthquake. Tectonophysics 491: 105-118.

Wang C Y, Han W B, Wu J P, Lou H and Chan W W (2007). Crustal structure beneath the eastern margin of the Tibetan Plateau and its tectonic implications. $J$ Geophys Res 112: B07307, doi:10.1029/2005 JB003873.

Wu J, Wang H and Cao J L (2011). Influence of crustal inhomogeneity on seismicity in North China. Chinese $J$ Geophys 54(8): 2 023-2 033 (in Chinese with English abstract).

Xie F R, Zhang Y Q and Zhang X L (2008). Estimation of Wenchuan $M_{\mathrm{S}} 8.0$ earthquake recurrence interval. Technology for Earthquake Disaster Prevention 3(4): 337-344 (in Chinese with English abstract).

Xu X W, Wen X Z, Chen G H and Yu G H (2008). Discovery of the Longriba fault in eastern of the Bayan Har block and its tectonic implications. Science in China (Series D) 38(5): 529-542 (in Chinese with English abstract).

Yamashina K (1978). Induced earthquakes in the Izu Peninsula by the Izu-Hanto-Oki earthquake of 1974, Japan. Tectonophysics 51: 139-154.

Zhang C J, Cao J L and Shi Y L (2008a). Viscosity of Tibet plateau in lower crust deduced from the postseismic deformation. Science in China (Series D) 38(10): 1 2501257 (in Chinese with English abstract).

Zhang C J, Shi Y L and Ma L (2009). Numerical simulation of crust rheological property reflected by post-seismic deformation of Kunlun large earthquake. Rock and Soil Mechanics 30(9): 2 552-2 558 (in Chinese with English abstract).

Zhang P Z, Xu X W, Wen X Z and Ran Y K (2008b). Slip rates and recurrence intervals of the Longmen Shan active fault zone, and tectonic implications for the mechanism of the May 12 Wenchuan earthquake, 2008, Sichuan, China. Chinese J Geophys 51(4): 1 066-1 073 (in Chinese with English abstract).

Zheng W J, Li C Y, Wang W T, Yin J H and Wei Z Y (2008). Trench logs of earthquake scarp of the $M_{\mathrm{S}} 8.0$ Wenchuan earthquake in the segment north of Beichuan. Seismology and Geology 30(3): 697-709 (in Chinese with English abstract).

Zhu J S (2008). The Wenchuan earthquake occurrence background in deep structure and dynamics of lithosphere. Journal of Chengdu University of Technology (Science and Technology Edition) 35(4): 348-356 (in Chinese with English abstract).

Zhu S B and Zhang P Z (2009). A study on the dynamical mechanisms of the Wenchuan $M_{\mathrm{S}} 8.0$ earthquake. Chinese J Geophys 52(2): 418-427 (in Chinese with English abstract). 